PROCEEDINGS OF THE

AMERICAN MATHEMATICAL SOCIETY

Volume 138, Number 5, May 2010, Pages 1657-1665

S 0002-9939(09)10104-1

Article electronically published on December 18, 2009

\title{
HAUSDORFF DIMENSIONS OF ESCAPING SETS OF TRANSCENDENTAL ENTIRE FUNCTIONS
}

\author{
LASSE REMPE AND GWYNETH M. STALLARD
}

(Communicated by Mario Bonk)

\begin{abstract}
Suppose that $f$ and $g$ are transcendental entire functions, each with a bounded set of singular values, and that $g \circ \varphi=\psi \circ f$, where $\varphi, \psi: \mathbb{C} \rightarrow \mathbb{C}$ are affine. We show that the escaping sets of $f$ and $g$ have the same Hausdorff dimension.

Using a result of the second author, we deduce that there exists a family of transcendental entire functions for which the escaping set has Hausdorff dimension equal to one.
\end{abstract}

\section{INTRODUCTION}

Let $f: \mathbb{C} \rightarrow \mathbb{C}$ be a transcendental entire function. The Julia set $J(f) \subset \mathbb{C}$ is the set of points where the family $\left(f^{n}\right)$ is not equicontinuous (with respect to the spherical metric). For an introduction to the dynamics of transcendental entire functions, see [Be1, Be2].

A number of authors have studied the Hausdorff dimension, $\operatorname{dim} J(f)$, of the Julia set (see [S6] for a survey). Baker [Bak] showed that $J(f)$ contains nontrivial continua for every transcendental entire function $f$, so in particular $\operatorname{dim} J(f) \geq 1$. Although it is known 55 that, for each $d \in(1,2]$, there are transcendental entire functions for which the Hausdorff dimension of the Julia set is equal to $d$, it is a well-known open question whether the Julia set of a transcendental entire function can have Hausdorff dimension equal to 1 .

More is known when we restrict the class of functions under consideration. The Eremenko-Lyubich class is defined by

$$
\mathcal{B}=\left\{f: f \text { is a transcendental entire function for which } \operatorname{sing}\left(f^{-1}\right) \text { is bounded }\right\},
$$

where $\operatorname{sing}\left(f^{-1}\right)$ consists of the critical and asymptotic values of $f$. The second author proved in $\mathrm{S3}$ ] that

$$
\operatorname{dim} J(f)>1 \quad \text { for } f \in \mathcal{B} .
$$

This proof in fact constructs a subset $A$ of the escaping set

$$
I(f):=\left\{z \in \mathbb{C}: f^{n}(z) \rightarrow \infty \text { as } n \rightarrow \infty\right\}
$$

Received by the editors April 20, 2009

2000 Mathematics Subject Classification. Primary 37F10; Secondary 37F35, 30D05.

Both authors are supported by the European CODY network. The first author is supported by EPSRC fellowship EP/E052851/1. 
whose closure $\bar{A}$ has Hausdorff dimension greater than one, which yields the desired conclusion due to the fact $[\mathrm{EL}$ ] that

$$
I(f) \subset J(f) \quad \text { for } f \in \mathcal{B} .
$$

It was shown more recently $[\mathrm{RS}$, that the set $I(f) \cap J(f)$ contains nontrivial continua for all transcendental entire functions, and hence

$$
\operatorname{dim}(I(f) \cap J(f)) \geq 1 .
$$

In view of these results, it seems natural to ask whether the escaping set of a transcendental entire function must have Hausdorff dimension greater than one. We show that this is not the case:

1.1. Theorem (Escaping sets of dimension one). There exists a function $f \in \mathcal{B}$ such that $\operatorname{dim} I(f)=1$.

Combined with previous results, this gives the following complete description of the possible Hausdorff dimensions of escaping sets.

1.2. Corollary (Dimensions of escaping sets). If $f$ is a transcendental entire function, then $\operatorname{dim} I(f) \in[1,2]$. Conversely, for every $d \in[1,2]$ there exists a function $f \in \mathcal{B}$ with $\operatorname{dim} I(f)=d$. If $d>1$, this function can be chosen such that $\operatorname{dim} J(f)=d$.

To prove Theorem 1.1 we consider a function that was studied in [S1. Let $L$ be the boundary of the region

$$
G=\{z: \operatorname{Re}(z)>0,-\pi<\operatorname{Im}(z)<\pi\},
$$

parametrized in the clockwise direction. Then

$$
F_{0}: \mathbb{C} \backslash \bar{G} \rightarrow \mathbb{C} ; \quad z \mapsto \frac{1}{2 \pi i} \int_{L} \frac{\exp \left(e^{t}\right)}{t-z} d t
$$

can be continued analytically to a transcendental entire function $F_{0}: \mathbb{C} \rightarrow \mathbb{C}$. From the properties of $F_{0}$ given in [S1], it can easily be seen that $F_{0} \in \mathcal{B}$.

Consider the family

$$
F_{\kappa}(z):=F_{0}(z)+\kappa, \quad \kappa \in \mathbb{C} .
$$

It is shown in [S1] that

$$
\lim _{\substack{\kappa \rightarrow-\infty \\ \kappa \in \mathbb{R}}} \operatorname{dim} J\left(F_{\kappa}\right)=1,
$$

while, by (1.1), $\operatorname{dim} J\left(F_{\kappa}\right)>1$ for all $\kappa \in \mathbb{C}$. This implies that $\operatorname{dim} J\left(F_{\kappa}\right)$ is a nonconstant function of $\kappa$.

In contrast, we show that the Hausdorff dimension of the escaping set cannot change in a family defined in this manner. More precisely, we say that two transcendental entire functions $f$ and $g$ are affinely equivalent if there are affine functions $\varphi, \psi: \mathbb{C} \rightarrow \mathbb{C}$ such that

$$
\psi \circ f=g \circ \varphi
$$

Any two functions $F_{\kappa_{1}}$ and $F_{\kappa_{2}}, \kappa_{1}, \kappa_{2} \in \mathbb{C}$, are clearly affinely equivalent. (Another well-known family consisting of transcendental entire functions that are affinely equivalent to each other is the family $z \mapsto \exp (z)+\kappa$ of exponential maps. Note that, for this family, the Julia sets and escaping sets all have Hausdorff dimension two by a result of McMullen $\mathrm{McM}$.) 
1.3. Theorem (Escaping dimensions and affine equivalence). Suppose that $f, g \in \mathcal{B}$ are affinely equivalent. Then $\operatorname{dim} I(f)=\operatorname{dim} I(g)$.

The proof uses recent results of the first author $[\mathrm{R}]$ on the rigidity of the dynamics near infinity for a function $f \in \mathcal{B}$. Using (1.1), (1.2) and (1.5), we obtain the following consequence of Theorem 1.3, which implies Theorem 1.1.

1.4. Corollary (Escaping dimension of $\left.F_{\kappa}\right)$. Let $F_{0}$ be the function defined above, and let $g \in \mathcal{B}$ be affinely equivalent to $F_{0}$. Then $\operatorname{dim} I(g)=1<\operatorname{dim} J(g)$.

Remarks.

(a) As far as we know, this provides the first example of an analytic family of entire functions for which the dimension of the escaping set is always strictly smaller than that of the Julia set.

(b) We note that if $f$ has finite order, then $I(f)$ has Hausdorff dimension two Bar, Sch, while for functions of "small" infinite order, a lower bound on the Hausdorff dimension of $I(f)$ is proved in BKS. It follows from these results that

$$
\limsup _{r \rightarrow \infty} \frac{\log \log \log \max |z|=r|f(z)|}{\log \log r}=\infty
$$

for any function with $\operatorname{dim} I(f)=1$. Note that for the maps $F_{\kappa}$ we have

$$
\log \log \log \max _{|z|=r}\left|F_{\kappa}(z)\right| \approx \log r .
$$

(c) For transcendental meromorphic functions, the Julia set may have any Hausdorff dimension $d \in(0,2]$ [S4. On the other hand, (1.1) was generalized to functions having a logarithmic singularity over $\infty$ in [BRS], and extended in BKZ to show that in fact the hyperbolic dimension of such a function is strictly larger than one. As far as we know, it is an open question whether the escaping set of a transcendental meromorphic function can have Hausdorff dimension zero; this cannot occur for the Julia set [S2]. Kotus and Urbański $[\mathrm{KU}$ ] have shown that there exist meromorphic functions with $\operatorname{dim} J(f)>\operatorname{dim} I(f)$; compare also [BK, Theorem 1.2].

(d) It is possible to prove Theorem 1.1 directly, using arguments similar to those in [S1].

To conclude our paper, we consider the notion of the eventual dimension of a transcendental entire function $f$, defined by

$$
\operatorname{edim}(f):=\inf _{R>0} \operatorname{dim} J_{R}(f),
$$

where, for each $R>0$,

$$
J_{R}(f):=\left\{z \in J(f):\left|f^{n}(z)\right| \geq R \text { for all } n \geq 1\right\} .
$$

Remark. This eventual dimension is also implicitly used by Bergweiler and Kotus BK] to obtain an upper bound for the Hausdorff dimension of the escaping set of certain meromorphic functions.

We show that for functions in the Eremenko-Lyubich class, the eventual dimension is an upper bound for $\operatorname{dim} I(f)$ and is also preserved under affine equivalence. This enables us to prove the following result. 
1.5. Theorem (Eventual dimensions of entire functions). For each $d \in[1,2]$, there exists a function $f \in \mathcal{B}$ such that $\operatorname{edim}(g)=\operatorname{dim} I(g)=d$ for all functions $g$ affinely equivalent to $f$.

Open questions. As far as we are aware, for all examples where $\operatorname{dim} I(f)$ and $\operatorname{edim}(f)$ are known, these numbers coincide. (This is also the case for the meromorphic functions considered in $[\mathrm{BK}$, Theorem 1.2].)

1.6. Question. Does there exist a function $f \in \mathcal{B}$ such that $\operatorname{edim}(f) \neq \operatorname{dim} I(f)$ ?

Following [EL, two entire functions $f$ and $g$ are called quasiconformally equivalent if there are quasiconformal functions $\psi, \varphi: \mathbb{C} \rightarrow \mathbb{C}$ such that (1.6) holds. Quasiconformal equivalence classes can be considered as natural parameter spaces. It was shown in $[\mathrm{R}]$ that, for $f \in \mathcal{B}$, the dynamical behaviour near infinity is the same for any function quasiconformally equivalent to $f$.

1.7. Question. Suppose that $f, g \in \mathcal{B}$ are quasiconformally but not affinely equivalent. Do $I(f)$ and $I(g)$ have the same Hausdorff dimension?

Remark. It follows from [ $\mathrm{R}$ that the answer is "yes" if $\operatorname{dim} I(f)=2$.

Structure of the article. In Section 2 , we use the results of $[\mathrm{R}]$ to deduce a fact that will play an important role in our proof of Theorem 1.3, which is itself proved in Section 3. We give the proof of Corollary 1.2 in Section 4 and treat eventual dimension and the proof of Theorem 1.5] in Section 5 .

\section{Conjugacies on $J_{R}(f)$}

We will require the fact (Corollary 2.2 below) that if two functions $f, g \in \mathcal{B}$ are affinely equivalent, then, for sufficiently large $R$, they are also quasiconformally conjugate on the set $J_{R}(f)$, and the dilatation of the conjugacy tends to one as $R \rightarrow \infty$. This follows from the ideas of $[\underline{R}$ but is not explicitly stated there. We shall provide a proof for completeness, using the following result, which is a special case of [ $\mathrm{R}$, Proposition 3.6].

2.1. Proposition (Existence of conjugacies). Let $f \in \mathcal{B}$. Let $\varphi_{\lambda}: \mathbb{C} \rightarrow \mathbb{C}, \lambda \in \mathbb{C}$, be a family of nonconstant affine maps that depend analytically on $\lambda$. Also suppose that $\varphi_{0}=$ id. We define $f_{\lambda}:=f \circ \varphi_{\lambda}$.

Let $N$ be a compact subset of $\mathbb{C}$ with $0 \in N$. Then there exists a constant $R>0$ such that, for every $\lambda \in N$, there is an injective function $\vartheta=\vartheta^{\lambda}: J_{R}(f) \rightarrow J\left(f_{\lambda}\right)$ with the following properties:

(a) $\vartheta^{0}=$ id,

(b) $\vartheta^{\lambda} \circ f=f_{\lambda} \circ \vartheta^{\lambda}$ and

(c) for fixed $z \in J_{R}(f)$, the function $\lambda \mapsto \vartheta^{\lambda}(z)$ is analytic in $\lambda$ (on the interior of $N$ ).

Remark. The conclusion of the theorem says that the injections $\vartheta^{\lambda}$ form a holomorphic motion of $J_{R}(f)$ on the interior of $N$ in the sense of Mañé, Sad and Sullivan. (Compare [H, Section 5.2].)

We now prove the main result of this section. 
2.2. Corollary (Conjugacy near infinity). Suppose that $f, g \in \mathcal{B}$ are affinely equivalent, and let $K>1$. Then there exist $R>0$ and a $K$-quasiconformal map $\vartheta: \mathbb{C} \rightarrow \mathbb{C}$ such that

for all $z \in J_{R}(f)$.

$$
\vartheta(f(z))=g(\vartheta(z))
$$

Proof. By conjugating $g$ with a Möbius transformation, we may assume for simplicity that $g=f \circ M$, where $M(z)=e^{A} z+B$ for suitable $A, B \in \mathbb{C}$. Define affine functions $\varphi_{\lambda}(z):=e^{\lambda A} z+\lambda B$ and consider the family

$$
f_{\lambda}(z):=f\left(e^{\lambda A} z+\lambda B\right) .
$$

Then $f_{0}=f$ and $f_{1}=g$.

We can apply Proposition 2.1 to $D:=(K+1) /(K-1)$ and $N:=\{\lambda:|\lambda| \leq D\}$ and obtain a number $R>0$ and maps $\vartheta^{\lambda}: J_{R}(f) \rightarrow J\left(f_{\lambda}\right)$ satisfying (国) to (ㄷ) as above.

It is well-known that a holomorphic motion such as $\vartheta^{\lambda}$ is quasiconformal as a function of $z$, and there is a bound on the dilatation of $\vartheta^{\lambda}$ in terms of the parameter $\lambda$. More precisely: if $\lambda \in \operatorname{int}(N)$, then $\vartheta^{\lambda}$ extends to a $K_{\lambda}$-quasiconformal map $\vartheta^{\lambda}: \mathbb{C} \rightarrow \mathbb{C}$ by $[\mathrm{BR}$, Theorem 1], where

$$
K_{\lambda} \leq \frac{D+|\lambda|}{D-|\lambda|}
$$

In particular, by the definition of $D$, the map $\vartheta_{1}$ extends to a $K$-quasiconformal map $\vartheta_{1}: \mathbb{C} \rightarrow \mathbb{C}$, as desired.

\section{Proof of Theorem 1.3}

Let $f$ be a transcendental entire function. For $R \geq 0$, we defined the set $J_{R}(f)$ in the introduction; let us also set

$$
I_{R}(f):=\left\{z \in I(f):\left|f^{n}(z)\right| \geq R \text { for all } n \geq 1\right\} .
$$

3.1. Lemma (Dimension and eventual dimension of escaping sets coincide). Let $f$ be a transcendental entire function and $R>0$. Then $\operatorname{dim} I(f)=\operatorname{dim} I_{R}(f)$ and $\operatorname{dim}(I(f) \cap J(f))=\operatorname{dim}\left(I_{R}(f) \cap J(f)\right)$.

Proof. Every point in $I(f)$ eventually maps to a point in $I_{R}(f)$, so we have

$$
I(f)=\bigcup_{j \geq 0} f^{-j}\left(I_{R}(f)\right),
$$

and analogously for $I(f) \cap J(f)$. The conclusion follows from the preservation of Hausdorff dimension under holomorphic functions and the countable stability of Hausdorff dimension.

Proof of Theorem 1.3. Suppose that $f, g \in \mathcal{B}$ are affinely equivalent. We want to show that $\operatorname{dim} I(f)=\operatorname{dim} I(g)$. By symmetry, it is sufficient to show that $\operatorname{dim} I(g) \geq \operatorname{dim} I(f)$. We shall use the following result, due to Gehring and Väisälä [GV, Theorem 8]: if $\vartheta$ is a $K$-quasiconformal map and $A \subset \mathbb{C}$, then

$$
\operatorname{dim}(\vartheta(A)) \geq \operatorname{dim} \vartheta(A) / K .
$$

(The optimal bounds on the distortion of Hausdorff dimension under quasiconformal mappings in dimension 2 are given by Astala's distortion theorem [A].) 
So, let $K>1$. It follows from Corollary 2.2 that there exist $R>0$ and a $K$-quasiconformal map $\vartheta: \mathbb{C} \rightarrow \mathbb{C}$ such that $\vartheta(f(z))=g(\vartheta(z))$ for all $z \in J_{R}(f)$.

For $z \in I_{R}(f) \subset J_{R}(f)$, we have

$$
g^{n}(\vartheta(z))=\vartheta\left(f^{n}(z)\right) \rightarrow \infty,
$$

and hence, by (3.1) and Lemma 3.1

$$
\operatorname{dim} I(g) \geq \operatorname{dim} \vartheta\left(I_{R}(f)\right) \geq \operatorname{dim}\left(I_{R}(f)\right) / K .
$$

Since $K$ was chosen arbitrarily close to 1 , this completes the proof.

\section{Proof of Corollary 1.2}

The first statement in Corollary 1.2 follows from (1.3). Recall that the second claim is that, for each $d \in[1,2]$, there exists a function $f \in \mathcal{B}$ for which the Hausdorff dimension of the escaping set is equal to $d$, and that furthermore if $d>1$, the function can be chosen such that $\operatorname{dim} J(f)=d$.

In the case $d=1$, this follows from Corollary 1.4, while for $d=2$ we can use $f(z)=\exp (z)$ and use McMullen's result [McM] that $\operatorname{dim} I(f)=2$. (In fact, by Bar, Sch, any entire function $f \in \mathcal{B}$ of finite order has the desired properties for $d=2$.)

The remaining cases are covered by the following statement, which is a consequence of the results of [S5] and [BKS].

4.1. Proposition (Escaping sets of dimension between one and two). For each $d \in(1,2)$, there exists a family of functions $f_{d, K}, K \in \mathbb{R}$, in the class $\mathcal{B}$ such that

$$
\operatorname{dim} J\left(f_{d, K}\right)=\operatorname{dim} I\left(f_{d, K}\right)=d .
$$

Proof. Set $p=(2-d) /(d-1)$ and define the family by

$$
f_{d, K}(z):=f_{p}(z)-K \quad \text { for } K \in \mathbb{R},
$$

where

$$
f_{p}(z):=\frac{1}{2 \pi i} \int_{L_{p}} \frac{\exp \left(e^{(\log t)^{1+p}}\right)}{t-z} d t
$$

with $L_{p}$ being the boundary of the region

$$
G_{p}=\left\{z=x+i y:|y| \leq \pi x /\left[(1+p)(\log (x))^{p}\right], x \geq 3\right\},
$$

described in the clockwise direction, for $z \in \mathbb{C} \backslash \overline{G_{p}}$. As with the function $F_{0}$ defined in the introduction, $f_{p}$ can be defined by analytic continuation for $z \in \overline{G_{p}}$.

It was shown in 55 that $f_{d, K} \in \mathcal{B}$ and that

$$
\operatorname{dim} J\left(f_{d, K}\right)=1+\frac{1}{1+p}=d
$$

for sufficiently large $K$. By (1.2), we have (again, for large $K$ ),

$$
\operatorname{dim} I\left(f_{d, K}\right) \leq \operatorname{dim} J\left(f_{d, K}\right)=d .
$$

On the other hand, the following result was proved in [BKS]. Suppose that $f \in \mathcal{B}, q \geq 1$, and that, for each $\varepsilon>0$, there exists $r_{\varepsilon}>0$ such that

$$
|f(z)| \leq \exp \left(\exp \left((\log |z|)^{q+\varepsilon}\right)\right) \quad \text { for }|z| \geq r_{\varepsilon} .
$$

Then $\operatorname{dim} I(f) \geq 1+\frac{1}{q}$. 
The functions $f_{d, K}$ satisfy the above assumptions for $q=1+p=1 /(d-1)$, and hence

$$
\operatorname{dim} I\left(f_{d, K}\right) \geq 1+\frac{1}{q}=d
$$

for all $K$. Together with (4.1), this implies that, for large $K$,

$$
\operatorname{dim} I\left(f_{d, K}\right)=\operatorname{dim} J\left(f_{d, K}\right)=d,
$$

as claimed.

\section{Eventual Dimension}

Recall that the eventual dimension of an entire function $f$ was defined in the introduction as

$$
\operatorname{edim}(f)=\inf _{R>0} \operatorname{dim} J_{R}(f) .
$$

We begin with the following two results.

5.1. Lemma. Let $f$ be a transcendental entire function. Then

$$
\operatorname{dim}(I(f) \cap J(f)) \leq \operatorname{edim}(f) \leq \operatorname{dim} J(f) .
$$

Proof. The second inequality holds by definition. On the other hand, by Lemma 3.1 we have

$$
\operatorname{dim}(I(f) \cap J(f))=\operatorname{dim}\left(I_{R}(f) \cap J(f)\right)=\operatorname{dim}\left(J_{R}(f) \cap I(f)\right)
$$

for all $R \geq 0$. Hence $\operatorname{dim}(I(f) \cap J(f)) \leq \operatorname{dim} J_{R}(f)$ for all $R \geq 0$, and thus we have $\operatorname{dim}(I(f) \cap J(f)) \leq \operatorname{edim}(f)$, as claimed.

5.2. Theorem. If $f, g \in \mathcal{B}$ are affinely equivalent, then $\operatorname{edim}(f)=\operatorname{edim}(g)$.

Proof. The proof is very similar to the proof of Theorem 1.3. Again, we need only show that $\operatorname{edim}(g) \geq \operatorname{edim}(f)$.

Let $K>1$. It follows from Corollary 2.2 that there exist $R>0$ and a $K$-quasiconformal map $\vartheta: \mathbb{C} \rightarrow \mathbb{C}$ such that

$$
\vartheta(f(z))=g(\vartheta(z))
$$

for all $z \in J_{R}(f)$.

Now let $S>0$ and choose $R^{\prime} \geq R$ sufficiently large that $|\vartheta(z)| \geq S$ whenever $|z| \geq R^{\prime}$. Then it follows from (5.1) that $\vartheta\left(J_{R^{\prime}}(f)\right) \subset J_{S}(g)$. Therefore, by (3.1) and Lemma 3.1.

$$
\operatorname{dim} J_{S}(g) \geq \operatorname{dim} \vartheta\left(J_{R^{\prime}}(f)\right) \geq \operatorname{dim} J_{R^{\prime}}(f) / K \geq \operatorname{edim}(f) / K .
$$

Since $K$ can be chosen arbitrarily close to 1 , we see that $\operatorname{dim} J_{S}(g) \geq \operatorname{edim}(f)$. Finally, since $S$ was arbitrary, it follows that $\operatorname{edim}(g) \geq \operatorname{edim}(f)$, as required.

We end this section by proving Theorem 1.5 ,

Proof of Theorem 1.5. Let $d \in[1,2]$. By Theorems 1.3 and [5.2, we only need to show that there is a function $f \in \mathcal{B}$ with $\operatorname{dim} I(f)=\operatorname{edim}(f)=d$. If $d>1$, then this follows from Corollary 1.2 together with (1.2) and Lemma 5.1. 
For $d=1$, we again consider the functions $F_{\kappa}, \kappa \in \mathbb{C}$, defined in the Introduction. It follows from (1.3), (1.5), Lemma 5.1 and Theorem 5.2 that

$$
\operatorname{edim}\left(F_{\kappa}\right)=1 \quad \text { for all } \kappa \in \mathbb{C} .
$$

Also, by Corollary 1.4 .

$$
\operatorname{dim} I\left(F_{\kappa}\right)=1 \quad \text { for all } \kappa \in \mathbb{C} .
$$

This completes the proof.

\section{REFERENCES}

A. Kari Astala, Area distortion of quasiconformal mappings, Acta Math. 173 (1994), no. 1, 37-60. MR.1294669 (95m:30028b)

Bak. I. Noel Baker, The domains of normality of an entire function, Ann. Acad. Sci. Fenn. Ser. A I Math. 1 (1975), no. 2, 277-283. MR0402044 (53:5867)

Bar. Krzysztof Barański, Hausdorff dimension of hairs and ends for entire maps of finite order, Math. Proc. Cambridge Philos. Soc. 145 (2008), no. 3, 719-737. MR2464786

BKZ. Krzysztof Barański, Bogusława Karpińska, and Anna Zdunik, Hyperbolic dimension of Julia sets of meromorphic maps with logarithmic tracts, Int. Math. Res. Not. IMRN 2009 (2009), no. 4, 615-624.

Be1. Walter Bergweiler, Iteration of meromorphic functions, Bull. Amer. Math. Soc. (N.S.) 29 (1993), no. 2, 151-188. MR.1216719 (94c:30033)

Be2. W Komplexe Dynamik, Lecture notes, fall 2008, http://analysis.math.unikiel.de/vorlesungen/dynamik.08/Gesamt.pdf.

BKS. Walter Bergweiler, Bogusława Karpińska and Gwyneth M. Stallard, The growth rate of an entire function and the Hausdorff dimension of its Julia set, J. London Math. Soc. 80 (2009), 680-698.

BK. Walter Bergweiler and Janina Kotus, On the Hausdorff dimension of the escaping set of certain meromorphic functions, preprint, 2009, arXiv:0901.3014.

BRS. Walter Bergweiler, Philip J. Rippon and Gwyneth M. Stallard, Dynamics of meromorphic functions with direct or logarithmic singularities, Proc. London Math. Soc. 97 (2008), no. 2, 368-400. MR2439666

BR. $\quad$ Lipman Bers and Halsey L. Royden, Holomorphic families of injections, Acta Math. 157 (1986), no. 3-4, 259-286. MR857675 (88i:30034)

EL. Alexandre È. Eremenko and Mikhail Yu. Lyubich, Dynamical properties of some classes of entire functions, Ann. Inst. Fourier (Grenoble) 42 (1992), no. 4, 989-1020. MR1196102 (93k:30034)

GV. Frederick W. Gehring and Jussi Väisälä, Hausdorff dimension and quasiconformal mappings, J. London Math. Soc. (2) 6 (1973), 504-512. MR0324028 (48:2380)

H. John H. Hubbard, Teichmüller theory and applications to geometry, topology, and dynamics. Vol. 1, Matrix Editions, Ithaca, NY, 2006. MR2245223 (2008k:30055)

KU. Janina Kotus and Mariusz Urbański, Hausdorff dimension of radial and escaping points for transcendental meromorphic functions, Illinois J. Math. 52 (2008), no. 3, 1035-1044.

McM. Curtis T. McMullen, Area and Hausdorff dimension of Julia sets of entire functions, Trans. Amer. Math. Soc. 300 (1987), no. 1, 329-342. MR.871679 (88a:30057)

R. Lasse Rempe, Rigidity of escaping dynamics for transcendental entire functions, Acta Math. 203 (2009), no. 2, 235-267.

RS. Philip J. Rippon and Gwyneth M. Stallard, Escaping points of meromorphic functions with a finite number of poles, J. Anal. Math. 96 (2005), 225-245. MR2177186 (2006f:30032)

Sch. Hendrik Schubert, Über die Hausdorff-Dimension der Juliamenge von Funktionen endlicher Ordnung, doctoral thesis, Christian-Albrechts-Universität Kiel, 2007, http://eldiss.uni-kiel.de/macau/receive/dissertation_diss_00002124.

S1. Gwyneth M. Stallard, The Hausdorff dimension of Julia sets of entire functions, Ergodic Theory Dynam. Systems 11 (1991), no. 4, 769-777. MR.1145621 (93e:30060)

S2. The Hausdorff dimension of Julia sets of meromorphic functions, J. London Math. Soc. (2) 49 (1994), no. 2, 281-295. MR1260113 (95b:58127) 
S3. - The Hausdorff dimension of Julia sets of entire functions. II, Math. Proc. Cambridge Philos. Soc. 119 (1996), no. 3, 513-536. MR1357062 (97a:58157)

S4. The Hausdorff dimension of Julia sets of meromorphic functions. II, J. London Math. Soc. (2) 60 (1999), no. 3, 847-859. MR1753818 (2001h:37096)

S5. - The Hausdorff dimension of Julia sets of entire functions. IV, J. London Math. Soc. (2) 61 (2000), no. 2, 471-488. MR.1760674 (2002b:37064)

S6. _ Dimensions of Julia sets of transcendental meromorphic functions, Transcendental dynamics and complex analysis, London Math. Soc. Lecture Note Ser., vol. 348, Cambridge Univ. Press, Cambridge, 2008, pp. 425-446. MR2458811

Department of Mathematical Sciences, University of Liverpool, Liverpool L69 7ZL, United KingDOM

E-mail address: 1.rempe@liverpool.ac.uk

Department of Mathematics and Statistics, The Open University, Walton Hall, Milton Keynes MK7 6AA, United Kingdom

E-mail address: g.m.stallard@open.ac.uk 